13 Ratten (BD-Stämme) erhielten einmal in der Woche $21 \mathrm{mg}$ DMH pro $\mathrm{kg}$ subcutan. Nach einer mittleren Indulstionszeit von $184+20$ Tagen und einer D 50 von $520+70 \mathrm{mg}$ pro kg starben alle Tiere mit multiplen Tumorknoten im Darm, meist an mehreren Stellen. Die Lokalisationen waren: Duodenum 6, Dünndarm 5, Colon 10 und Rectum 8 Fälle (Fig. 1). Die histologische Diagnose (THomas) ergab Adenocarcinome mit infiltierendem Wachstum. Weitere Versuche mit wöchentlich einmal $7 \mathrm{mg}$ pro $\mathrm{kg}$ subcutan und $21 \mathrm{mg}$ pro $\mathrm{kg}$ oral ergaben bisher bei 20 Ratten die gleichen Tumoren. Lokale Sarkome an der Injektionsstelle wurden nicht beobachtet. Damit ist die experimentelle Erzeugung von Darmkrebs möglich. Daß dies gerade durch subcutane Injektion gelang, beweist die Selektivität der Wirkung. Die völlige Verschiedenheit der carcinogenen Wirkung von Dimethyl- und Diaethylhydrazin zeigt, daß die Art der Alkyl-Reste die Organotropie entscheidend verändern kann. Diese Frage wird systematisch untersucht, ebenso der Wirkungsmechanismus.

Herrn Dozent Dr. C. Thomas, Pathologisches Institut in Bonn, danken wir für die histologischen Untersuchungen, der Deutschen Forschungsgemeinschaft für großzügige Förderung.

Eingegangen am 26. Januar 1967

[1] Drvekrey, H., R. Preussmann, F. Matzkies u. S. Ivankovic: Naturwissenschaften 53, 557 (1966). - [2] Druckrey, H., R. Preussmann, S. Ivankovic, C. H. Schmidt, B. T. So u. C. ThoMAS: $Z$. Krebsforsch. 67, 31 (1965). - [3] DrUCKREY, H., R. PREvSSMANN, D. SCHNÄHL u. M. MUELLER: Naturwissenschaften 48, 134 (1961). - DruCkrey, H., R. Preussmann, S. Ivankovic u. D SchmämL: Z. Krebsforsch. (im Druck). - [ [ ] Laqueur,.G.: Virchows Arch. 340, $151(1965 / 66)$.

\section{Über eine Reaktion von Lysozym mit Gangliosiden}

\section{G. UHLENBRUCK}

Max-Planck-Institut für Hirnforschung, Abteilung für Tumorforschung und experimentelle Pathologie, Köln

Vor kurzem berichteten wir über die Hemmung der PolylysinErythrozytenaggregation duxch Ganglioside [1]. Inzwischen hat Grossgerbauer [2] gefunden, daß das basische Enzym Lysozym ebenfalls Erythrozyten aggregiert. Nach unseren Untersuchungen an verschiedenen Blutkörperchen entfaltet sich dabei keine enzymatische Wirkung. Dieses gut wasserlösliche und leicht (kommerziell) erhältliche Protein schien uns als „Ersatz" für Polylysin geeignet. Bei vergleichenden Experimenten stellte sich nun heraus, daß Lysozym in der Agargel-Diffusion unter scharfer Präzipitatbildung mit Gangliosiden reagiert (Fig. 1).
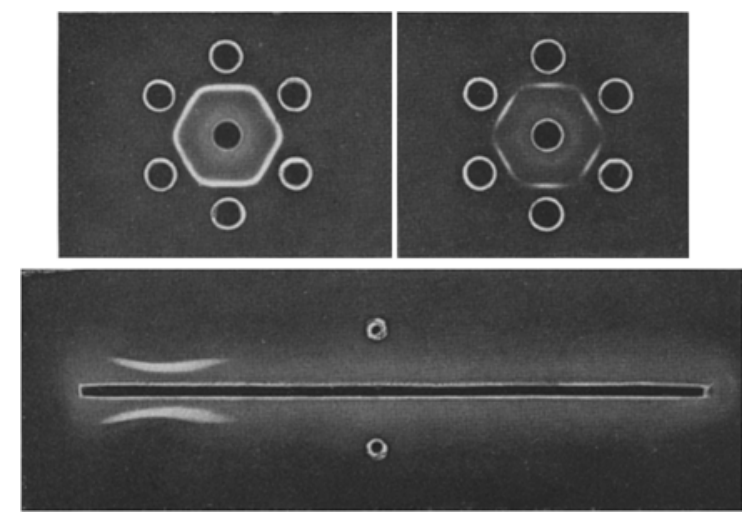

Fig. 1. Agargel-Diffusion zwischen einem Gangliosid-Gemisch (Gehirn) und Lysozym. Mittelloch bzw. Graben: $10 \%$ Lysozym hirn) und Lysozym. Mittelloch bzw. Graben: $10 \%$ Lysozym
(Lights \& Co.); AußBenlöcher: $10 \%$ (oben links und Bild unten) (Lights \& Co.); Außenlöcher: $10 \%$ (oben links und Bild unten)
bzw. $5 \%$ Gangliosid-Lösung. Unteres Bild: links Kathode, Methode nach SCHEIDEGGER [3] (Immunelektrophorese)

Obwohl es sich um ein Gemisch von Gangliosiden handelt tritt in der Regel keine Trennung ein und nur eine Präzipitatlinie auf. Diese Wechselwirkung zwischen einem basischen Protein (Lysozym) einerseits und den sauren Gruppen der Ganglioside andererseits ist insofern von Bedeutung, da im Zentralnervensystem neben Gangliosiden ein basisches Protein vorkommt, das man als wirksames Antigen bei der aller- gischen, experimentell erzeugten Encephalomyelitis erkannt hat. Die Frage, ob in vivo Beziehungen zwischen basischen Proteinen und Gangliosiden eine Rolle spielen [4], sei dahingestellt. - Der Befund weist außerdem auf eine mögliche Fehierquelle bei der Bestimmung von Antikörpern gegen Ganglioside hin. Interessanterweise haben wir eine solche Präzipitation zwischen Lysozym und neuraminsäurehaltigen $\mathrm{Mu}$ coiden bisher nicht beobachtet. Mithin ist es wahrscheinlich, daß u. a. Ganglioside bei der Erythrozytenaggregation durch Lysozym eine nicht unbedeutende Rolle spielen, denn Erythrozytenganglioside zeigen das gleiche Phänomen.

Ich danke der DFG für die Unterstützung der Arbeit, Herra Priv.Doz. Dr. W. Grelen für Proben gereinigter Ganglioside, FrI. UTE REIFENBERG für ihre geschickte Hilfe und Frl. EDITH KLASMEIER für die Anfertigung der Fotos.

Eingegangen am 30. Januar 1967

[1] UHLENBRUCK, G.: Naturwissenschaften 53, 505 (1966). [2] GrossGerauER, K.: Blut 14, 93 (1966). - [3] SCHEIDEGGER, J. J.: L'immunélectrophorèse. Semaine hôp. 32, 2119 (1956). [4] MCCluer, J. F.: Vortrag $X^{\text {th }}$ Int. Congr. Biochem. Lipids, Köln 1966.

\section{DC-Potentiale der Hirnrinde}

O. VAtTer und B. Rischmaul

Max-Planck-Institut für Hirnforschung,

Physiologische Abteilung, Göttingen

Die Schädeloberfläche von Mensch und Tier weist gegenüber einem indifferenten Punkt ein positives DC-Potential (Ruhepotential) auf [1], dessen Maximum über der regio praecentralis liegt. Dort fanden sich beim Kaninchen epidural +6 bis $+9 \mathrm{mV}$, mit einem negativen Gradienten zur Schädelhaut hin: Das äußere Periost war meist noch positiv, die Hautoberfläche häufig negativ. Kleine Tiere (Mäuse) gaben kleinere, der Mensch größere DC-Potentiale. Bei Messung mit ElektrolytTiefenelektroden schlägt das DC-Potential etwa in der Mitte der Hirnrinde, beim Kaninchen 1,2-1,5 $\mathrm{mm}$ subpial, ins Negative um und erreicht Werte bis $-14 \mathrm{mV}$, die in größerer Tiefe wieder langsam abnehmen. Beim Zurückziehen der Elektrode tritt 1-1,2 mm subpial der Umschlag $z$ u positiven DC-Werten auf. Durch Infusion isotonischer NaCl-Lösung in den Cortex kann das DC-Potential auf $10-20 \%$ absinken. Schwankungen des gegen die Haut abgeleiteten DC-Potentials rühren hauptsächlich von pH-Schwankungen infolge veränderter Hautdurchblutung her. Es wird angenommen, daß das DC-Potential durch gliöse Membranpotentiale $[2,3] \mathrm{zu}-$ stande kommt, deren Größe von der $\mathrm{K}^{+}$-Konzentration im Interzellulärspalt abhängt. Deshalb nimmt das DC-Potential bei Erweiterung der Interzellulärspalten infolge Infusion von $\mathrm{NaCl}-$ Lösung ab. Die unterschiedliche Größe der DC-Spannung in verschiedenen Rindenfeldern weist auf die Bedeutung der histologischen Struktur hin (größere Grenzflächen Neuronensoma-Glia im Bereich der Riesenpyramidenzellschicht). Als Stromkreis, der das negative Tiefen- und das positive Oberflächenpotential verursacht, käme eine $\mathrm{K}^{+}-\mathrm{A}$ bwanderung aus der erregungsreichen [4] tiefen Rinde mit Räckflu $\mathrm{G}$ der positiven Ladung über die Glia in Betracht. Das beträchtliche Volumen $\left[5,6^{6}\right]$ und der geringe Ohmsche Widerstand der Interzellulärflüssigkeit lassen erwarten, daß nur etwa die Hälfte des Stromkreiswiderstands auf den interzellutären Leitungsweg entfällt. Da die DC-Potentiale dieselbe Umkehrung in der Tiefe zeigen wie evozierte Rindenpotentiale, sind ursächliche Zusammenhänge zwischen evoziertem Potential und DC-Potential denkbar. Die Dipoltheorie [y] scheint uns die Potentialinversionen nicht zu erklären, da unsere kapazitiven Ableitungen zum Nachweis der Dipolpotentiale quantitativ ebensowenlig befriedigten wie die Ergebnisse unserer induktiven Ableitungen [8].

Eingegangen am 19. Januar 1967

[1] Rischmaur, B., u. O. Vatter: Pflüger's Arch. ges. Physiol. 291, 27 (1966). - [2] KuFFLER, S. W., I. G. NICHOLIS u. R. K. ORKAND J. Neurophysiol. 29, 768 (1966). - [3] ORKand, R. K., I. G. NICHOLLS u. S. W. KUFFLER: J. Neurophysiol. 29, 788 (1966).

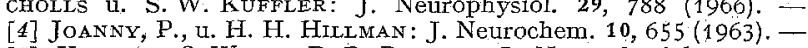
[5] Kuffles, S. W., u. D. D. PotTer: J. Neurophysiol. 27, 290 (1964). - [6] NicholLS, I. G., u. S. W. KuFfLER: J. Neurophysiol. $28,519(1965) .-[7]$ BURNS, B. D.: The mammalian cerebral cortex. London: Edward Arnold 1958. - [8] NIEDNER, N., u. O. VATTER: Pflügers Arch. ges. Physiol. 291, 31 (1966). 